\section{OHP-023 DIFFERENCES IN TRAINING REQUIRED FOR HOSPITAL PHARMACY PRACTISE IN FRANCE AND QUEBEC}

doi:10.1136/ejhpharm-2013-000276.397

'A Guérin, D Merger, E Courbon, ME Métras, D Lebel, JF Bussières. CHU SainteJustine, Pharmacy, Montreal, Canada

Background During a one-year internship in a Quebec teaching hospital, a group of French pharmacy interns explored the similarities and differences in training.

Purpose To compare the training required for hospital pharmacy practise in France and in Quebec.

Materials and Methods This is a descriptive comparative study. A list of relevant themes was established by consensus after a review of key websites and literature. A panel of three French interns, a Quebec hospital pharmacy resident and two teaching hospital pharmacists was assembled. Similarities and differences for each theme were identified and discussed.

Results Twenty-seven themes were selected with seven similarities and twenty differences between France and Quebec. In both countries, post-graduate training included a selection process, a structured programme with pre-identified topics, lectures and experiential courses. While post-graduate training is perceived as a plusvalue, it is not mandatory. Amongst the differences identified, the two post-graduate systems have been offered for a different period of time (1815-France vs. 1961-Quebec), French interns are not working as pharmacists while Quebec residents are, French internship lasts 4 years vs. 16 months in Quebec, French annual scholar fees are lower (500 euros/year vs. 3840 euros/18 months in Quebec), both programmes offers two paths (hospital/industry in France; hospital/community pharmacy in Quebec), French internship locations includes healthcare agencies, laboratories, research units, hospitals while Quebec residency focuses on patient care locations in hospitals/retail pharmacy and admission capacity differs. Other differences were identified in geographic mobility, resident status, obligations and responsibilities, modalities of supervision, compensation, on-call shifts and evaluation.

Conclusions There are significant differences between French and Quebec post-graduate training although both require work in hospital settings. A better understanding of these similarities and differences may contribute to reciprocal improvement of these programmes and favour exchanges between the two countries.

No conflict of interest.

\section{OHP-024 DOSES OF ANTI-TUMOR NECROSIS FACTOR IN CLINICAL PRACTISE: A FOUR-YEAR RETROSPECTIVE STUDY IN ANKYLOSING SPONDYLITIS PATIENTS}

doi:10.1136/ejhpharm-2013-000276.398

'V Escudero-Vilaplana, '2E Ramírez-Herraiz, 'N Trovato-López, ${ }^{2} E$ Alañón-Plaza, ${ }^{1}$ MJ Bellini, ${ }^{1}$ A Herranz-Alonso, ${ }^{2}$ A Morell-Baladrón, 'M Sanjurjo-Sáez. ${ }^{1}$ Hospital General Universitario Gregorio Marañón, Pharmacy, Madrid, Spain; ${ }^{2}$ Hospital Universitario La Princesa, Pharmacy, Madrid, Spain

Background Achieving minimum clinically effective doses could improve the efficiency of treatment with anti-TNF.

Purpose To evaluate the mean dose in ankylosing spondylitis (AS) patients treated with adalimumab (ADA), etanercept (ETN) or infliximab (IFX) in clinical practise and to estimate mean patient-year costs.

Materials and Methods Observational, multicentre, retrospective study performed in two tertiary hospitals. AS patients who received ADA, ETN or IFX from October 2006 to October 2010 were included. Patients could constitute several cases if they received different sequential treatments for at least 6 months. Mean drug consumption was analysed based on individual hospital pharmacy service claims. Demographic data, C-reactive protein (CRP), HLA-B27, axial or mixed AS subtypes, disease activity (BASDAI, BASFI) and concomitant and previous AS treatments were analysed. Associated costs were estimated based on public ex-factory prices including tax (2011 Euros). IFX cost included $€ 110.93$ per infusion.

Results 119 patients were included, for a total of 137 cases. No differences were found in recorded variables among groups, except fewer IFX patients $(8.2 \%$ ) had previously received a biological treatment than ETN $(25.0 \%)$ or ADA $(28.6 \%)$ patients ( $<<0.05)$.

ANCOVA and multivariate regression analysis showed that the only variable to affect patient-year costs was anti-TNF treatment (table 1).

Conclusions Although IFX patients started with a basal PCR lower than ADA patients and a basal BASFI lower than those treated with ETN, no differences were found among groups at the end of the study. IFX doses were higher than ETN doses as a percentage of the label doses.

\section{Abstract OHP-024 Table 1}

\begin{tabular}{llll}
\hline & ADA & ETN & IFX \\
\hline Cases & 28 & 48 & 61 \\
Basal CRP (mg/dl) & $2.00^{*}$ & 1.46 & 0.83 \\
Final CRP (mg/dl) & 0.40 & 0.57 & 0.92 \\
Basal BASFI & 5.1 & $5.3^{*}$ & 3.7 \\
Final BASFI & 3.7 & 3.7 & 4.0 \\
\% patients achieving BASDAl $<4$ & $60.0 \%$ & $60.5 \%$ & $58.3 \%$ \\
Patient-year cost (label doses) & $€ 12,860$ & $€ 11,846$ & $€ 13,928$ \\
Study mean doses (\% of label doses) & $37.12 \mathrm{mg} / \mathrm{biw}$ & $44.39 \mathrm{mg}$ weekly & $5.1 \mathrm{mg} / \mathrm{kg} / 8 \mathrm{wk}$ \\
& $(92.80 \%)$ & $(88.78 \%)^{*}$ & $(101.99 \%)$ \\
Patient-year cost (study clinical & $€ 11,934^{*}$ & $€ 10,516^{*}$ & $€ 14,235$ \\
practise doses) & & &
\end{tabular}

" $p<0.05$ vs. IFX

No conflict of interest.

\section{OHP-025 DRUG INFORMATION AND THE USE OF A PILLBOX TO IMPROVE SATISFACTION OF PATIENTS TREATED WITH TEMOZOLOMIDE}

doi:10.1136/ejhpharm-2013-000276.399

I Escribano Valenciano, JM Caro Teller, I Cañamares Orbis, C García Muñoz, M Campo Angora, JM Ferrari Piquero. Hospital 12 de Octubre, Hospital Pharmacy, Madrid, Spain

Background Compliance is sometimes difficult for patients treated with temozolomide, because of the inconvenience due to the high daily number of capsules needed. Studies with other drugs showed that pillboxes increased patient satisfaction.

Purpose To determine if pharmaceutical information and the use of pillboxes may improve satisfaction of patients treated with temozolomide.

Materials and Methods This prospective and interventional study included adult patients who picked temozolomide up in our Hospital Pharmacy (01/03/2012 to 31/08/2012).

In the first visit, patients previously treated with temozolomide completed a satisfaction questionnaire, which was adapted from the ESTAR questionnaire (ARPAS study). It consisted of 9 questions to be answered from 0 (very unsatisfied) to 6 (very satisfied), and another two items about temozolomide information. In addition, pharmaceutical information and pillboxes were provided to all patients.

At their next visit, patients received another questionnaire, with 6 of the previous satisfaction questions and 5 new questions about usefulness of the pillbox and of the received information.

Results 35 patients were evaluated with the first questionnaire $(50.69 \pm 13.38$ years old; $77.14 \%$ were treated with $\geq 3$ capsules per dose) and 28 of them filled in the second questionnaire (50.32 \pm 12.45 
years old; $75 \%$ taking $\geq 3$ capsules per dose). $88.57 \%$ vs. $85.71 \%$ of patients took their pills in cycles of 5 days followed by 23 days without treatment.

Satisfaction pre- and post-intervention was related to: the number of capsules prescribed per dose $(4.43 \pm 1.60 \mathrm{vs}$. $4.96 \pm 0.84)$, the possibility of taking their treatment everywhere $(5.17 \pm 0.92$ vs. $5.32 \pm 0.82)$, and the convenience of the chemotherapeutic regime $(5.06 \pm 0.94$ vs. $5.07 \pm 1.05)$.

The usefulness of the pharmaceutical attention, the pillbox and the leaflet were valuated as $5.46 \pm 0.58,5.39 \pm 0.69$ and $5.68 \pm 0.48$, respectively. Global satisfaction with pharmaceutical attention was $5.79 \pm 0.42$.

Conclusions In this study, information provided by hospital pharmacist and the use of pillboxes improved satisfaction in patients treated with temozolomide.

No conflict of interest.

\section{OHP-026 ECONOMIC EVALUATION OF ANTIFUNGAL DRUGS IN AN INTENSIVE CARE UNIT}

doi:10.1136/ejhpharm-2013-000276.400

${ }^{1} \mathrm{~F}$ Cattel, ${ }^{2} \mathrm{M}$ Chiumente, ${ }^{3} \mathrm{~F}$ Ancona, ${ }^{3} \mathrm{~A}$ Miliacca, ${ }^{4} \mathrm{FG}$ De Rosa, 'M Scaldaferri, 'E Sciorsci, 'F Re, 'S Stecca. 'A.O.U. Città della Salute e della Scienza, Hospital Pharmacy, Turin, Italy; ${ }^{2}$ University of Turin, School of hospital pharmacy, Turin, Italy; ${ }^{3}$ PricewaterhouseCooper Advisory s.p.a., Pharmaceutical \& Life Sciences, Turin, Italy; ${ }^{4}$ C.O. Amedeo di Savoia-Birago di Vische, Amedeo di Savoia Hospital, Turin, Italy

Background Anidulafungin is a semisynthetic echinocandin, mainly used in invasive Candida infections in non-neutropenic patients, with a daily dose cost lower than other antifungal drugs used in candidiasis treatment.

Purpose To prepare a mathematical model, able to produce an estimate of the savings that could be realised using anidulafungin instead of the other antifungals.

Materials and Methods A pilot study was carried out at Turin hospital 'Città della Salute e della Scienza', involving two Intensive Care Units (ICUs), which are the major consumers of echinocandins.

In these two ICUs:

- Data concerning consumption, prices and 2010 rebates for various antimycotics were collected;

- The medical records of 174 patients, admitted in 2010, were examined to identify all those instances where anidulafungin could have been appropriately used, instead of other antifungals.

Based on the analysis of medical records, the substitution index of the other antimycotics with anidulafungin has enabled us to calculate its potential use and the saving that the hospital might be able to achieve.

Results The analysis revealed a frequently inappropriate use of various antifungal drugs.

The review of medical reports confirmed a $70 \%$ substitution index of liposomal amphotericin B with anidulafungin.

In 2010, the hospital used 9,237 vials of caspofungin, anidulafungin and liposomal amphotericin B.

If we assume $100 \%$ use of anidulafungin in instances where it would be appropriate in the two ICUs, the hospital could make savings exceeding Euro 100,000 per year.

Results The ICUs in question account for $18 \%$ of the total vials. The possible savings that could be made by extending the analysis and application of the mathematical model to the entire hospital have not yet been investigated, but the model has confirmed the initial assumption of possibly saving money by using anidulafungin, according to approved indications, in substitution for other antimycotic drugs.

No conflict of interest.

\section{OHP-027 EDUCATIONAL MODEL FOR IT SYSTEMS}

doi:10.1136/ejhpharm-2013-000276.401

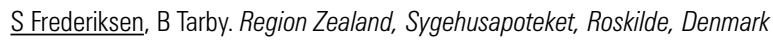

Background An electronic documentation management system had been implemented, replacing and uniting three previously paper-based documentation systems. 130 users located on 6 different locations needed to be educated in the new system.

Purpose To evaluate the training on the new system. Would it be possible for a large number of employees at 6 different locations to use an educational model, called Less Is More (LIM) to rapidly learn a new IT system, with a high degree of satisfied users?

Materials and Methods The training of the personnel was carried out by educating 30 supervisors over a period of 5 days. The supervisors conducted the education of the remaining personnel, based on the principles and materials of LIM. The education model (LIM), is based on simple graphic displays with very simple drawings, that do not require e.g. PowerPoint. The graphic displays give a visual overview of the system. The graphic displays are supplemented by a short storey, telling the procedure of the system. In addition the drawings are followed up by a single page, navigation note with short instructions on how to carry out a specific procedure.

The evaluation was performed using a user survey of the course. The users were to complete a questionnaire, rating relevant statements on a scale from extremely satisfactory, very satisfactory, satisfactory, less than satisfactory and not satisfactory.

Results A survey conducted after the course showed that all 30 supervisors found the method extremely satisfactory, very satisfactory or satisfactory. 100 users on 6 different locations were subsequently educated in a period of 4 weeks, and reports from the system show that the system is being used as desired.

Conclusions We would recommend LIM as an educational model for IT systems, it was used successfully with a high degree of satisfied users.

No conflict of interest.

\section{OHP-028 EFFECT OF A CLOSED SYSTEM DEVICE AND NEW CLEANING PROCEDURE ON SURFACE CONTAMINATION WITH CYTOSTATICS}

doi:10.1136/ejhpharm-2013-000276.402

ZS Órás, I Higyisán, M Kis Szölgyémi. Bajcsy-Zsilinszky Hospital, Pharmacy, Budapest, Hungary

Background The potential for contamination associated with handling cytostatic drugs exists in the workplace despite compliance with the protective measures for the safe handling of cytostatics.

Purpose To investigate the efficacy of using closed system drug transfer devices and implementing a new cleaning procedure for environmental cytostatics contamination in the central cytostatics department.

Materials and Methods Wipe samples were taken from five defined areas in March, 2011: Laminar air flow (LAF) cabinet, workbench, floor in front of the LAF cabinet, transport box and the handle of the refrigerator located in the make-ready room. They were tested for contamination with 8 substances (5-Fluorouracil (5-FU), cyclophosphamide (CP), ifosfamide (Ifos), gemcitabine (Gem), etoposide (Eto), methotrexate (MTX), pacli-taxel (Pac), docetaxel (Doc)) using LC-MS/MS. After seven months the test was repeated on the same surfaces (except the refrigerator handle) after the implementation of PhaSeal closed-system drug transfer device and $0.1 \mathrm{~m} \mathrm{NaOH}$ decontamination solution.

Results In the first test the level of substances wiped from the refrigerator handle was under the detection limit. The LAF cabinet 